\title{
Procedure for Creating Personalized Geometrical Models of the Human Mandible and Corresponding Implants
}

\author{
Karim Najm HUSAIN, Miloš STOJKOVIĆ, Nikola VITKOVIĆ, Jelena MILOVANOVIĆ, Miroslav TRAJANOVIĆ, Mohammed RASHID, \\ Aleksa MILOVANOVIĆ
}

\begin{abstract}
The greatest challenge in engineering of human mandible implants lies in its customization for each patient individually, by adapting them to the patient's anatomical, morphological and physiological characteristics. This customization maximizes the efficiency of the patient's health recovery process. The application of anatomically shaped and personalized bone endoprosthesis, fixation plate and scaffold models bring great improvement to the clinical practice in maxillofacial surgery. It ensures that implant meets the biomechanical and dentofacial aesthetic requirements and, ultimately, reduces complications during recovery. In order to create such implants, novel procedure based on personalized models of mandible and its parts, and also plates and scaffold implants is presented in this paper. Design procedures for the creation of the personalized models are based on the application of Method of Anatomical Features, which has been already applied for the creation of geometrical models of human bones. This procedure improves pre-surgical planning, enables better execution of surgical intervention, and as a consequence improves patient recovery processes.
\end{abstract}

Keywords: anatomical features; fixation plate; mandible implant; scaffold; personalized model

\section{INTRODUCTION}

Advances in the field of reconstructive surgery, engineering, material science, information technology additive manufacturing and other scientific disciplines, enabled researchers to develop various solutions and to find new procedures and techniques for rebuilding bones or bone parts which are exposed to trauma, and to personalize it to the specific patient. The major goal of the mandible (lower jaw) reconstruction is to enable its proper functioning, provide the required support to the outer soft tissue, accelerate healing, address psychological damage and facilitate the quality of postoperative life $[1,2]$. The first step in rebuilding processes of mandible is creation of geometrically accurate and anatomically correct geometrical model of the bone $[3,4]$. The geometrical models which conform to the anatomy and morphology of the mandible can be created using volumetric imaging methods (e.g. Computerized Tomography - CT or Magnetic Resonance Imaging - MRI), 2D methods (X-ray, 2D Ultrasound), and predictive methods, which are based on predictive models. Volumetric methods provide 3D models which can be used for measuring morphometric parameters and initial placement of implants using medical software (e.g. Materialize Mimics) [4]. These types of models do not have proper geometrical definitions and correlations between anatomical entities. They do not have the ability to change and adapt to various requirements which may be: to quickly change position of the screw axis in order to maintain the adequate stability of the assembly, to define geometrical properties and type of the contact surface of the implant, to proper position the bone fragments, to create changeable FEA model based on the contact geometry between implant and bone, etc. Predictive methods enable creation of bone geometrical models by using various types of parametric (statistical) models. This method can provide valid geometrical models, but they are limited by the input set of the bone samples, applied method, and by the number and type of the parameters involved [5,6]. As already stated, mandible bone can be subjected to fracture or some other type of trauma, e.g. tumor. Depending of the fracture type or the size of the affected or missing part of the bone, for its proper rebuilding, plate implants and/or scaffolds can be used. In general, plates can be divided into two general groups: Locking plates and Non-locking plates [7-10]. Locking plates provide better stability of the bone-plate assembly and does not require pre-contouring of the plates. Non-locking plates require pre-contouring, and they can interrupt and destroy the periosteum of the bone [10]. In both cases it is important to properly adjust shape and position of the plate(s) in accordance with mandible geometry. Scaffolds are structures that have been designed to enable cellular interactions to contribute to the formation of new tissue, and they provide a structural support for tissue development. Scaffolds can be used in various medical situations, especially in clinical cases when affected part of the mandible bone is relatively large. In order to recreate damaged part of mandible, an accurate geometric model of the implanted scaffold can be designed by the application of CAD software. This software can be used for the creation of unit cell 3D geometrical model that matches the complex outer shape of the lost segment and simulates a more complex internal structure. This model should enable: good permeability for the new growing cells, cells proliferation, migration and passage of nutrients, etc. [1114].

Main goal of this research was to define complete procedure for the creation of personalized implants which can be used in the treatment processes of the mandible trauma. This procedure enables creation of the mandible, plate and scaffold personalized geometrical and physical models. For that purpose, Method of Anatomical Features (MAF) $[5,15]$ is applied for the creation and definition of the mandible geometry and morphology, and also for the definition of the shape and geometry of the scaffold implant and fixation plate. This procedure and included models can be used for the treatment of mandible fractures classified by the AO/OTA classification [16-18] and for the reconstructive surgery of mandible parts affected by some kind of disease (e.g. tumors, osteoporosis). Support goals of the research were to provide orthopedic surgeons ability to adequately prepare and perform orthopedic interventions. All of the methods and procedures which 
were applied to achieve the defined goals are presented in this paper.

The main research idea was to develop improved procedure for the implant creation, which will enable surgeons to perform orthopedic interventions with much more anatomical, morphological and geometrical accuracy. The one of the possible ways to improve orthopeadic and maxillofacial surgical intervention, especially ones that heal large trauma, which is characterized by missing part of the bone (complex fractures and tumors), is to develop and apply personalized implants. The one aspect of implant personalization that is very important for bone treatment is geometrical personalization. Geometric personalization is related to creating of implants that are geometrically congruent to the bone shape of a particular patient, that is, to the specific anatomy of the patient's traumatized bone region. The implants that are mainly use in orthopaedics are fixation elements (e.g. plates), endoprosthesis (e.g. for hip or knee) and latterly bone scaffolds. Besides the geometrical personalization, in orthopaedics, personalization of implant should include biomechanical suitability and, of course, bio-chemical compatibility. However, this research was focused just on the procedure for creating of geometrically personalized implant assembly, which is consisted of a bone scaffold and fixation plate. In addition, as for verification case, we chose large trauma of mandible because this kind of traumas are often [2] and it is usually characterized with high aesthetic demands, which additionally underline importance of anatomical personalization of an implant assembly in today's clinical practice.

\section{THE PROCEDURE}

The main process is described by using Structured Analysis and Design Technique (SADT) notation. SADT uses diagrams to describe process functionality [19]. The basic elements of SADT are: input elements, resources, control elements, output elements and various types of arrows and connection elements [19]. The process of implants customization is presented in Fig. 1, and in SADT notation it is defined as A0 process with the following elements:

- Input elements: medical image of the patient, parametric models of the mandible fracture and fixators

- Control elements: anatomical knowledge about human bones, medical image analysis knowledge, anatomical and morphological rules, rules defined in MAF

- Resources: doctor, designer, software packages (Medical imaging software, CATIA)

- Output elements: geometrical model(s) of the customized implants (plate and scaffold).

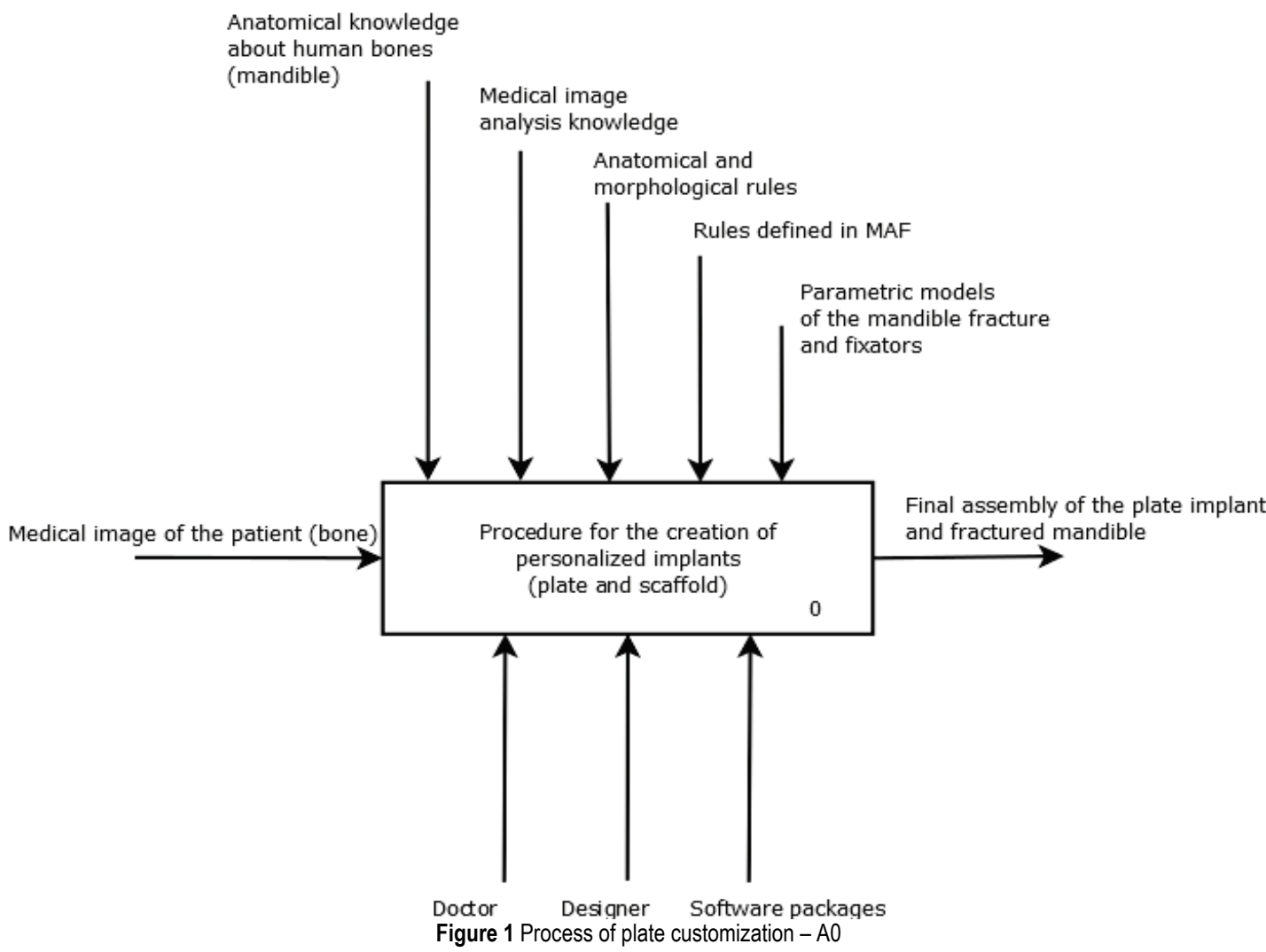

The A 0 process is defined in more detail and described in A1 diagram presented in Fig. 2. In this diagram whole procedure for the creation of the customized fixator is described. The procedure can be split into individual operation which defines suitable activities:
- Analysis of the medical image acquired from CT scanner or X-Ray. Doctors (radiologist, orthopedists, etc.) use radiological images (computer tomography images are preferable) to determine type of fracture, its status and direction, then to determine which kind of scaffold should be implanted and finally what fixation 
plate should be applied to attach the fractured parts of lower jaw. Important sub-activity within this analysis is morphometry, i.e. capturing the values of specific morphometric parameters, which are already established for mandible and defined in [5]. These values are measured by using morphometry capturing tools of the applied Medical Imaging Processing (MIP) software. These parameters are essential for digital reconstruction of the geometric model that is sufficiently congruent, i.e. personalized to the "healthy" shape of the patient's mandible (geometry without fracture or affected part). Furthermore, by controlling the geometric model of the bone, these parameters directly affect the geometry of all associated models that are built out of the "healthy" bone geometric model: geometric model of the fracture (trauma) and models of applicable implants: the fixation plate and the scaffold. Thus, the output from this analyzing activity may be defined as "Collected knowledge about mandible fracture" as it is noted in Fig. 2.

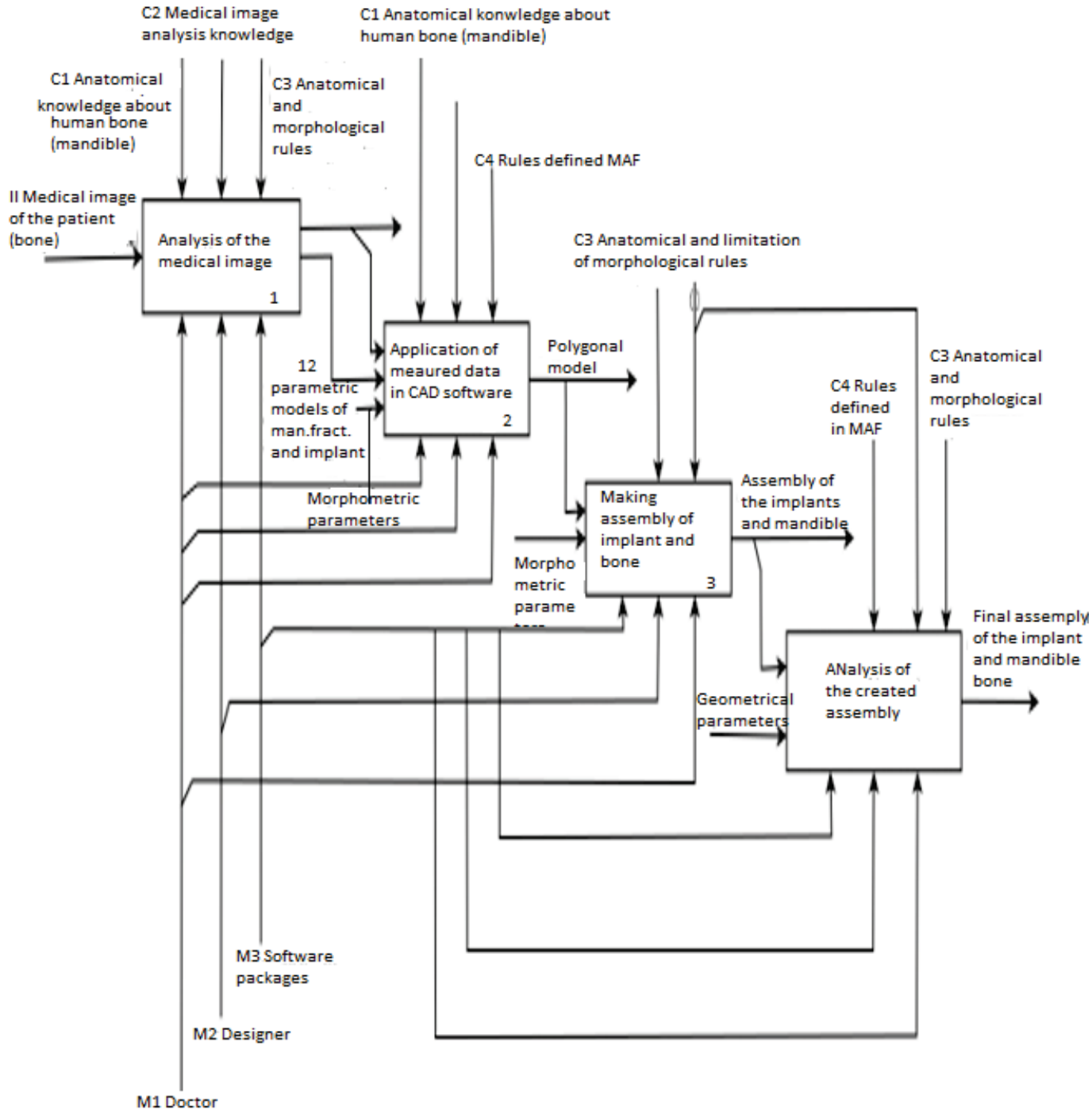

Figure 2 Complete process diagram - A1

- Application of the measured data in CAD. The values of morphmetric parameters that are measured from the scans of a particular patient are used to conduct the modification of generic parametrically driven polygonal 3D model generating personalized 3D polygonal model of "healthy" mandible. The generic polygonal model of the lower jaw, which is harnessed by a set of morphometric parameters, should be created previously [5]. Due to the nature of the polygonal CAD model, it is possible that resulting models can have some geometry and topological errors. Yet, these errors can be fixed by applying proper CAD features, such as correction and optimization of the model in a sense of number of triangles, orientation of triangles, triangles reduction, filling the holes, etc. These corrections or so-called modelhealing operations are very important because only topologically regular and closed polygonal models can be later converted into the solid models that are necessary for creating the implant assembly. Having the personalized 3D polygonal model of "healthy" mandible as a base model, the next sub-activity is digital reconstruction of geometry of traumatized region, fracture or tumor affected bone. The boundary surfaces of the fracture or affected bone part are defined also by the morphometric measurements conducted through radiological images analysis. Finally, parametric models of the fixation plate and 
scaffold are being designed in accordance with the defined type of fracture, that is, with the affected bone part. At the end of this process, as an outcome, the sound polygonal models are created and prepared for the other activities.

- Making assembly of bone and implants - Within this activity, polygonal models of the impaired jaw bone and traumatized region are being converted into the solid models by applying corresponding CAD features and after that, an assembly of the injured mandible, fixation plate(s) and scaffold is being created

- Analysis of the created assembly - In this activity, the focus is on determining of deviation between "healthy" and reconstructed geometry of the injured jaw bone linked within the assembly (that is, how it is expected to be healed). This analysis encompasses anatomical, morphological, and geometrical aspects of deviations. Within the anatomical aspect, analysis should determine whether all the anatomical entities important for the proper positioning of the implants are there. Similarly, morphological aspect of analysis inspects whether all prominent shapes of the anatomical entities are preserved. Calculation of geometrical deviations between "healthy" and reconstructed model of the jaw bone indicates the achieved geometrical accuracy level. Tolerated deviations should be minimal, but still in practice these are determined by surgeon's experience, that is, by applying the rule of thumb. If all the deviations are within the tolerated limits, then the first iteration of procedure for creating of personalized geometrical model of the assembly of injured bone, fixation plate(s) and scaffold is finished, and the model is ready for further considerations like simulating biomechanics, surgical intervention preparation and analysis of manufacturability.

\section{METHOD OF ANATOMICAL FEATURES (MAF)}

In order to implement this kind of procedure, it is of great importance to create parametric model of the mandible ensuring associative propagation of geometric changes towards the models of the mandible traumatized region and the implants (plates and scaffolds). The underlying method used for the creation of parametric 3D model of jaw bone is a kind of modification of MAF [5], the method which is the result of our previous work, and has been already implemented and proved as efficient for the creation of the 3D models of other human bones. As a result of the method application, a different type of geometrical models of specific bone can be created (e.g. parametric, polygonal, surface, etc.). These models are based on the Referential Geometrical Entities (RGEs), which are created individually for every bone in accordance with the bone anatomy, morphology and morphometry, and serve as basic geometry (lines, points, planes, axis, etc.) used for models creation. Parametric model is an output from MAF application, and it is defined by a set of parametric functions, which represent coordinates of carefully defined points on bone outer surface. These points can be anatomical points, i.e. defined on already established anatomical landmarks of the bone, or points which are carefully selected to improve model generation. Parametric functions are created by the application of statistical methods on the set of input bone samples. In order to customize (personalize) a model to the individual patient, values of morphometric parameters are obtained from patient's medical images, and applied in parametric functions $[5,6,15]$.

Lower jaw (mandible) is the biggest face bone, which is linked with skull through the temporomandibular joint, and it consists of body, two rami, two coronoid and two condylar process as presented in [17, 18]. Mandible body looks similar to horseshoe shape and it lies in horizontal plane. It has external and internal side and two edges, alveolar part of the mandible which matches the inferior dental arc and lower edges or mandible foundation. Ramus is approximately of a quadric shape which is located upward and backward in relation to mandible body. It forms an angle of $90^{\circ}-140^{\circ}$ to the mandible body, and it has external and internal side, four edges, upper, lower, anterior, and posterior. On the upper edge two processes are located: coronoid process and condylar process.

\subsection{Developed Parametric Models}

There are four important parametric models which were created, and they are: parametric model of the mandible, parametric models of the mandible fractures, parametric model of the scaffold, and parametric model of the generic mandible fixation plate implant. In the following section, the procedures for the creation of corresponding models of the mandible fractures, scaffold and plate implant will be explained with the application of the sample mandibles acquired from the University Clinical Center of Nis. The same mandible samples were used, as ones defined in [5].

\subsection{Mandible Fracture Parametric Model}

Mandible fractures are classified according to the AO/OTA classification system [16], and they can be arranged by levels. The level 1 as the very primary CMF classification defines existence of mandibular fractures, irrespective of their description in location, number, pattern, or morphology. Level 2 classification system [20], groups minimal criteria for the rating and registration of CMF fractures, allowing the characterization of the fracture topography within defined regions based on the examination of diagnostic X-rays and/or CT imaging (Fig. $3)$. The more detailed level 3 classification system [21] (dentition, atrophy, fracture morphology) is presented in [22]. It is also important to note that each fracture can be simple or complex, as is presented in Fig. 3 and Fig. 4.

The main idea for creating geometrical models of mandible fractures is to use specific anatomical points which have been already defined for the mandible personalized point cloud. As it is already stated, product of MAF application is a personalized point cloud model for the specific bone, i.e. in this case, mandible.

In order to define geometrical model of the fracture, proper points can be selected on the personalized point cloud model, as presented in Fig. 5. The "line" or "lines" of fracture is being modeled as NURBS(es) by interpolating the spline curves over the selected points (Fig. 5). In the case of large trauma or tumor, where a part of the mandible 
is missed, the missed volume of the bone has to be subtracted from the rest of the bone volume and in this CAD operation the spline curves of the fracture are used as cutting boundary references (Fig. 6).

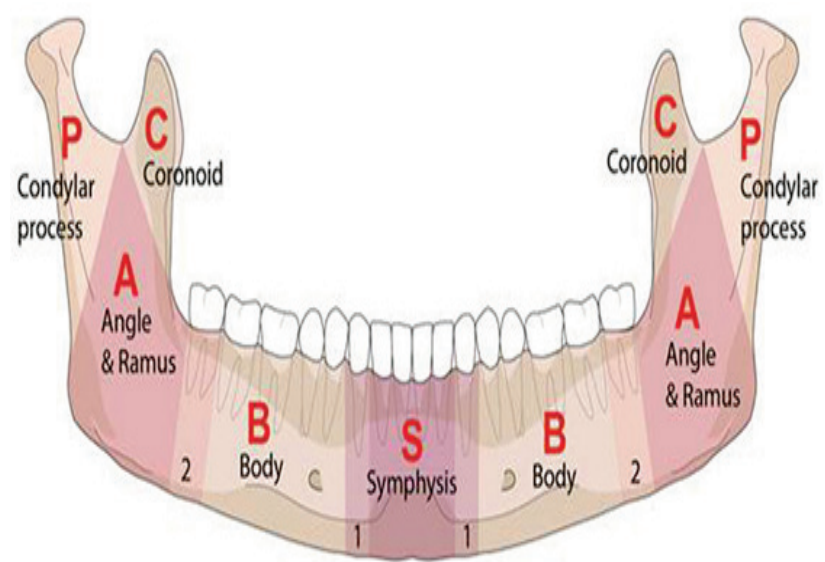

Figure 3 Mandible with layout of anatomical regions and transitional zones
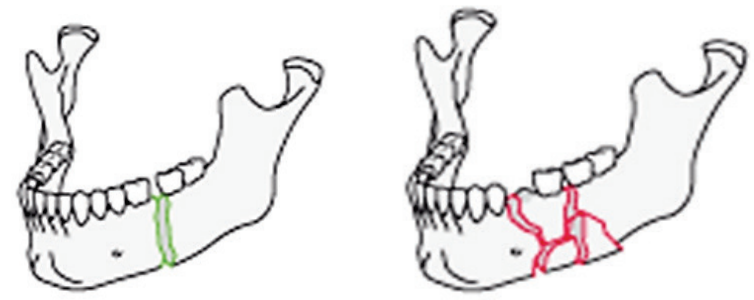

Figure 4 Mandible body fracture simple and complex

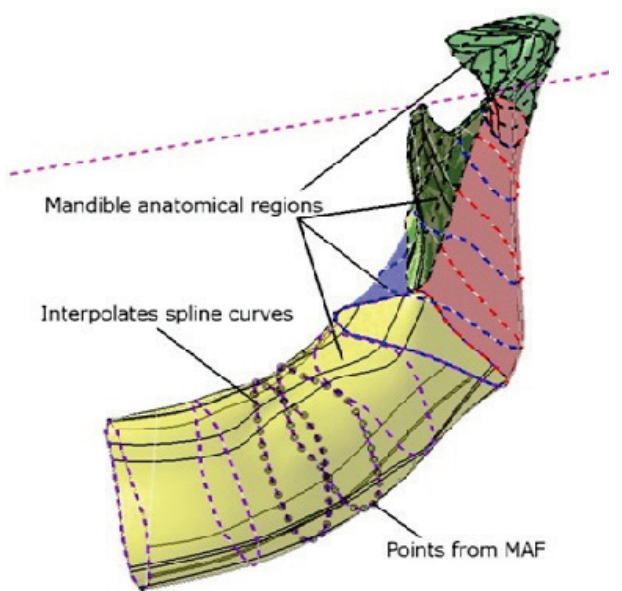

Figure 5 Mandible anatomical regions together with interpolated splines

At the same time, the volume of the missed part defines the 3D boundary surface (an envelope) for bounding (or bordering) the model of the scaffold which is going to be designed and placed (together with bone graft) in the space of fracture. It is clear that the most important step in the process of fracture modeling is to define adequate reference points. There are two possible ways of creating fracture models based on reference points. First, fracture model can be created in advance by creating a database of fracture models defined by OTA classification [20, 21]. These models are represented by NURBS curves defined by using parametrically defined points. Each group of points which belongs to the individual NURBS curve is defined as vector with an index, and stored. By using these vectors, NURBS curve for particular fracture can be created. Second, geometrical model of the fracture can be created by directly selecting points and creating NURBS curves on the personalized cloud model of the mandible. The latter is applied in here, and the former will be applied in the future work.

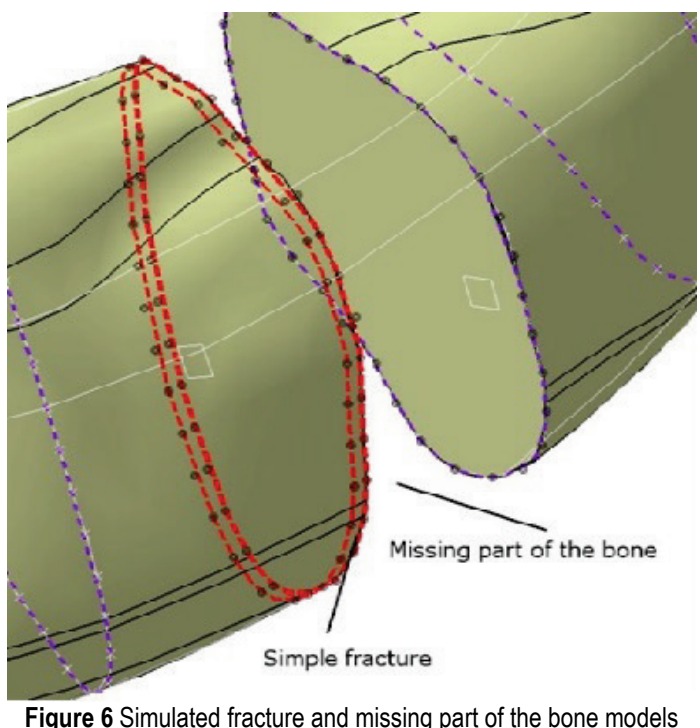

\subsection{Parametric Model of Plate Implants}

Today, fixation plates which are mainly applied in bone fixation and reduction are ones capable for both locking and non-locking functions, such are Locking Compression Plates (LCP). LCP provide better fixation and they can withstand more load compared to standard Dynamic Compression Plates (DCP) [8-10]. DCP and LCP fixation methods are based on anatomically pre-contoured plates, reducing or eliminating intra-operative plate bending. LCP does not require accurate contouring, because, that is not required in cases when locking screws are used. In such clinical situations plate acts more like fixator rod. However, greater distance between the plate and the bone can cause a problem [10]. It is important to mention another plate type commonly applied for mandible fractures. These plates are called reconstructive plates. They are designed with profound notches among the holes, and they can be contoured in three planes to fit surfaces. Reconstruction plates are provided in straight and slightly thicker and stiffer pre-curved lengths. They have oval screw holes, like mentioned compression plates, and they allow potential limited compression [22, 23].

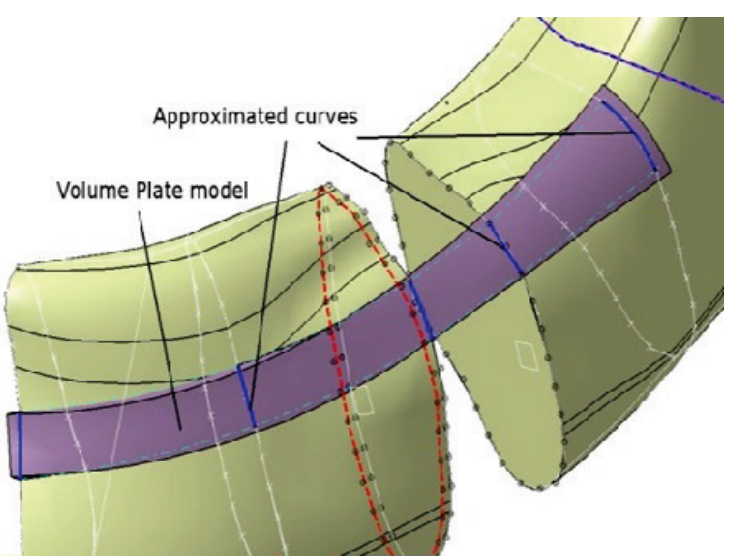

Figure 7 Personalized plate model created by using mandible surface model 
To create so-called personalized geometrical models of the plates, which fit anatomy of the traumatized region of the mandible, there is a need to use geometrical model of the patient's mandible as a base model that shape of plate should follow [24]. Thus, the very first step is to digitally reconstruct the model of mandible's boundary surface. This step starts with importing cloud of points that is gained by the computer tomography image (scans) and finishes with mandible boundary surface model sculpturing and tuning of its parameters. The next step is creation of the spatial curves that will define boundaries of the fixation plates. These curves are generated as NURBS that pass through the points, which lie on the boundary surface of the mandible model, as presented in Fig. 7. At the region of trauma, where a bone part misses, i.e. where fracture exists, these curves are interpolated ensuring the minimum of continuity in $\mathrm{U}$ and $\mathrm{V}$ direction. The model of the plate is being created by using adequate number of the approximate curves created over the just a few selected points of interpolated curves as presented in Fig.8. In this way, the parameters that control the mandible boundary surface propagate their influence on the fixation plate geometry model. The volume of the fixation plate model is being created by using multi-section technical feature with approximate curves as sections (Fig. 8). This approach enables creation of plate model which goes through whole mandible, specific mandible region, or specific number of regions (Fig. 7) [25].

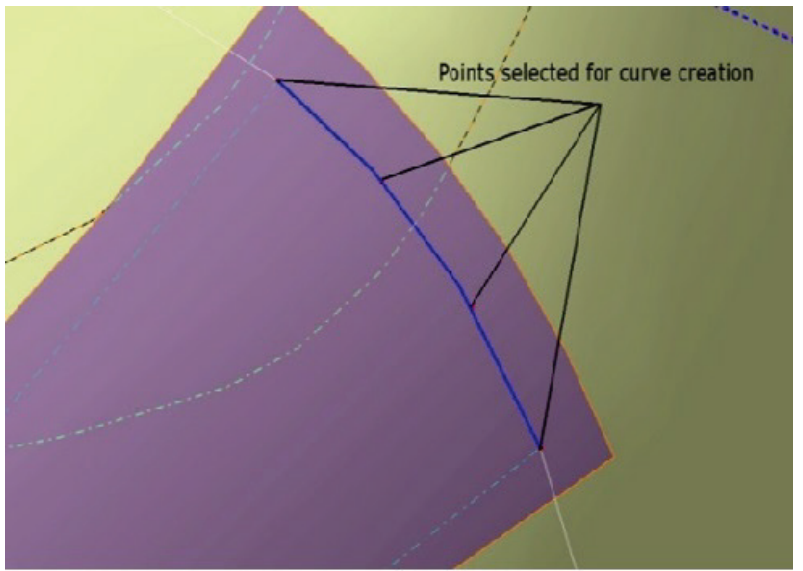

Figure 8 Personalized plate model created by using mandible surface model, closer look on points selected for curve creation

Hence, the shape and geometry of the fixation plate model can be controlled by the input points (personalized point cloud) or by additional points created on the mandible surface model. The presented approach enables creation of various types of fixation plate, controlling the geometry of the model in the same time. If accurate precontouring is required, more points on mandible surface can be selected and more precise plate model will be created.

\subsection{Anatomically Bordered Scaffold Implant}

Mandible bone can be subjected to trauma, and because of that, a part of the bone can be missing, or disabled to perform its function(s). This is specifically the case when bone is affected by tumor. Surgeon needs to remove that part of the bone in order to improve patient's health status. In order to accelerate bone regeneration processes, this missing part of the bone can be replaced by implantation of the bone macro-scaffold. To avoid misunderstanding regarding usage of a term of tissue scaffold, we decide to use the term of "macro-scaffold" that should point out a kind of reinforcement framework structure, which is far larger than intracellular matrix structures, and which should provide mechanical support for the bone graft during its transformation from the prototissue to mature tissue and its interconnecting with neighboring tissue, and, finally, in that way, to help recovery process of the missing tissue. Scaffolds are usually produced by the application of additive manufacturing, in which object is manufactured on the basis of developed 3D geometrical model. Because of that, to create geometrical model of a scaffold that is personalized to the particular patient's bone anatomy, it is important to anatomically shape or border the scaffold design. It is preferable to follow the shape of the bone in order to protect the surrounding tissue of damage caused by scaffold. In order to achieve that goal, specific design approach for the creation of scaffold geometrical model is applied. The approach is based on using 3D model of scaffold that is consisted of unit cell elements multiplied in $3 \mathrm{D}$ space, in order to create the $3 \mathrm{D}$ pattern of unit cell shapes that fills the volume of the missing part of the bone [6], as presented in Fig. 9.

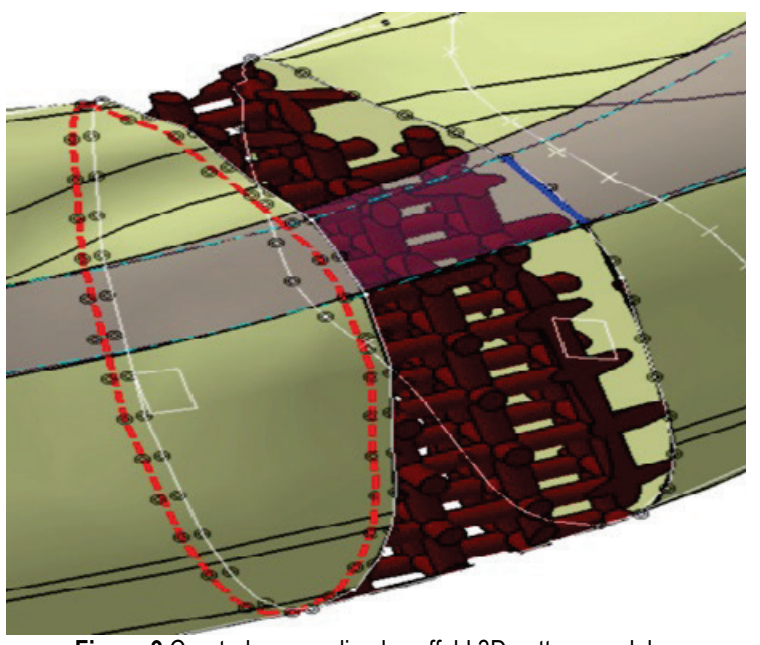

Figure 9 Created personalized scaffold 3D pattern model-m

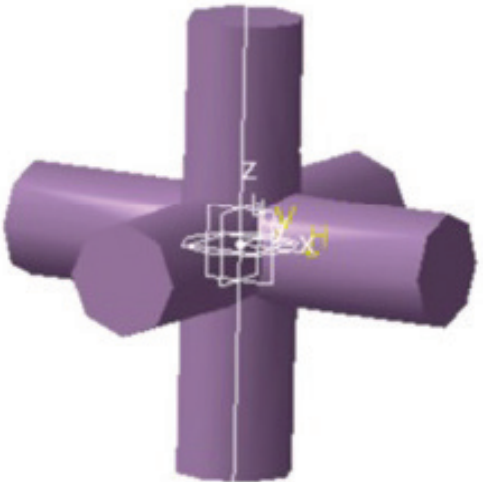

Figure $103 \mathrm{D}$ volume model of the cylindrical unit cell element

Unit cell design can vary [26], but in this research cylindrical design is applied and presented in Fig. 10. The dimension of the unit (cells elements) is determined by 
suitable value of manufacturing dimension of applied additive technology and chosen material (e.g. for titanium, or some other biodegradable polymer minimal wall thickness is $0.5 \mathrm{~mm}$ ). The scaffold was created by the application of rectangular pattern technical feature on the unit cell model. The final scaffold model is made by cutting the scaffold model with boundary surface of the missing part of the bone (Boolean subtract operation), and it is presented in Fig. 9.

We should put a word about possibility to control pattern density and shape and in that way to control mechanical properties of the scaffold (elasticity, i.e. stiffness). This should be the theme for some other paper, but, still, we should mention here that controlling the shape and density of unit cells in the pattern may be used for biomechanical optimization (e.g. to ensure proper deflection of the structure that may initiate or keep ossification process).

\section{CONCLUSION}

The paper introduces novel procedure which can be implemented for digital geometry modelling of an implant assembly that is aimed for personalized bone tissue recovery treatment. It is consisted of a bone macroscaffold, which is anatomically bordered according to the particular patient mandible, and from the fixation plates that are also anatomically curved to the particular patient mandible shape. Defined approach includes application of design procedures which enable creation of personalized models of implants (scaffold and plate) and mandible bone. Design procedures are based on the application of MAF method and parametric bone models developed by its application. The main benefits are for surgeons and patients, but the developed models and procedures can be also used in education of medical students and practitioners. Students and medical practitioners can use these models to improve their techniques and knowledge in surgery planning and simulation. Future work presumes creation of automated procedures applied in user defined feature (UDF) created in CAD software. This UDF should provide more intuitive user interface for the surgeons, students, or medical practitioners, because it will enable faster preparation or simulation of surgical interventions.

\section{Acknowledgements}

The paper presents the case that resulted from application of the research projects id III 41017 (Virtual Human Osteoarticular System and its Application in Preclinical and Clinical Practice) and TR35006, supported by the Ministry of Education, Science and Technological Development of the Republic of Serbia.

\section{REFERENCES}

[1] Olson, R.A., Fonseca, R.J., Zeitler, D.L., Osbon, D.B. (1982). Fractures of the mandible: a review of 580 cases. Journal of Oral and Maxillofacial Surgery, 40(1), 23-8. https://doi.org/10.1016/S0278-2391(82)80011-6

[2] Ellis, E. 3rd, Moos, K.F., el-Attar, A. (1985). Ten years of mandibular fractures: an analysis of 2,137 cases. Oral Surg Oral Med Oral Pathol., 59(2), 120-9.

https://doi.org/10.1016/0030-4220(85)90002-7
[3] Van Eijden, T.M. (2000). Biomechanics of the mandible. Crypt Rev Oral Biol Med., 11(1), 123-36. https://doi.org/10.1177/10454411000110010101

[4] Benazzi, S., Stansfield, E., Kullmer, O., Fiorenza, L., \& Gruppini, G. (2009). Geometric morphometric methods forbone reconstruction: the mandibular condylar process of Pico della Mirandola. Anat Rec (Hoboken), 292(8), 10881097. https://doi.org/10.1002/ar.20933

[5] Vitković, N., Mitić, J., Manić, M., Trajanović, M., Husain, K., Petrović, S., \& Arsić, S. (2015).The Parametric Model of the Human Mandible Coronoid Process Created by Method of Anatomical Features. Computational and Mathematical Methods in Medicine. Article ID 574132. https://doi.org/10.1155/2015/574132

[6] Vitkovic, N., Stojkovic, M., Majstorovic, V., Trajanovic, M., $\&$ Milovanovic, J. (2018). Novel design approach for the creation of 3D geometrical model of personalized bone scaffold. CIRP Annals, 67(1), 177-180. https://doi.org/10.1016/j.cirp.2018.04.064

[7] Kurakar, M. (2014). Comparison of Stability of Fracture Segments in Mandible Fracture Treated with Different Designs of Mini-Plates Using FEM Analysis. J Maxillofac Oral Surg., 13(3), 310-319. https://doi.org/10.1007/s12663-013-0510-y

[8] Gellrich., N. C., Suarez-Cunqueiro, M. M., Otero-Cepeda, X. L., Schön, R., Schmelzeisen, R., \& Gutwald, R. (2004). Comparative study of locking plates in mandibular reconstruction after ablative tumor surgery: THORP versus UniLOCK system. Journal of Oral and Maxillofacial Surgery, 62(2), 186-193. https://doi.org/10.1016/j.joms.2003.04.012

[9] Herford, A. S. et al. (1998). Use of a locking reconstruction bone plate/screw system for mandibular surgery. Journal of Oral and Maxillofacial Surgery, 56(11), 1261-1265. https://doi.org/10.1016/S0278-2391(98)90605-X

[10] Haug, R. H., Street, C. C., \& Goltz, M. (2002). Does plate adaptation affect stability? A biomechanical comparison of locking and onlocking plates. Journal of Oral and Maxillofacial Surgery, 60(11), 1319-1326. https://doi.org/10.1053/joms.2002.35732

[11] Polo-Corrales, L., Latorre-Esteves, M., \& Ramirez-Vick, E. J. (2014). Scaffold Design for Bone Regeneration, $J$. Nanosci. Nanotechnol, 14(3), 15-56. https://doi.org/10.1166/jnn.2014.9127

[12] Chen, G., Ushida, T., \& Tateishi, T. (2002). Scaffold Design for Tissue Engineering, Macromol. Biosci, 2, 67-77. https://doi.org/10.1002/1616-5195(20020201)2:2<67::AIDMABI67>3.3.CO;2-6

[13] Bose, S., Vahabzadeh, S., \& Bandyopadhyay, A. (2013). Bone tissue engineering using 3D printing. Materials Today, 16(12), 496-504. https://doi.org/10.1016/j.mattod.2013.11.017

[14] Laurencin, C., Khan, Y., \& El-Amin, S. F. (2006). Bone graft substitutes. Expert Rev Med Devices, 3(1), 49-57. https://doi.org/10.1586/174344440.3.1.49

[15] Majstorovic, V., Trajanovic, M., Vitkovic, N., Stojkovic, M. (2013). Reverse engineering of human bones by using method of anatomical features. CIRP Annals Manufacturing Technology, 62(1), 167-170. https://doi.org/10.1016/j.cirp.2013.03.081

[16] AO Foundation, https://aotrauma.aofoundation.org, AO/OTA classification, last access: 15.07.2018.

[17] Lipski, M., Tomaszewska, I. M., Lipska, W., Lis, G. J., \& Tomaszewski, K. A. (2007). The mandible and its foramen: anatomy, anthropology, embryology and resulting clinical implications. Folia Morphol., 72(4), 285-292. https://doi.org/10.5603/FM.2013.0048

[18] Chakraborty, S. K. (2007). Management of Bilateral Condylar Fractures: Case Review. Med J Armed Forces India. 63(1), 85-87.

https://doi.org/10.1016/S0377-1237(07)80123-7 
[19] Fahim Ahmed, A. (2015). Using structured analysis and design technique (SADT) for simulation conceptual modelling. Doctoral Thesis, Loughborough University. https://doi.org/10.1109/WSC.2014.7019963

[20] Kunz, C., Audigé, L., Cornelius, C. P., et al. (2014). The Comprehensive AOCMF Classification System: Midface Fractures - Level 2 Tutorial. Craniomaxillofacial Trauma \& Reconstruction, 7(1), 59-67. https://doi:10.1055/s-0034-1389560

[21] Cornelius, C. P., Audigé, L., Kunz, C., Rudderman, R., Buitrago-Téllez, C. H., Frodel, J., \& Prein, J. (2014). The Comprehensive AOCMF Classification System: Mandible Fractures-Level 3 Tutorial. Craniomaxillofacial Trauma \& Reconstruction, 7(1), 31-43. https://doi.org/10.1055/s-0034-1389559

[22] Harjani, B., Singh R. K., Pal, U. S., \& Singh, G. (2012). Locking $\mathrm{v} / \mathrm{s}$ non-locking reconstruction plates in mandibular reconstruction. Natl J Maxillofac Surg. 3(2), 159-165. https://doi.org/10.4103/0975-5950.111371

[23] Guk-Jin, S., Eun-Gyu, J., Jong-Sung, L., So-Young, C., JinWook, K., Tae-Geon, K., \& Jun-Young, P. (2014). Reconstruction plates used in the surgery for mandibular discontinuity defect. J Korean Assoc Oral Maxillofac Surg. 40(6), 266-271. https://doi.org/10.5125/jkaoms.2014.40.6.266

[24] Gutwald, R., Jaeger, R., \& Lambers, F. M. (2017). Customized mandibular reconstruction plates improve mechanical performance in a mandibular reconstruction model, Comput Methods Biomech Biomed Engin. 20(4), 426-435. https://doi.org/10.1080/10255842.2016.1240788

[25] Vitkovic, N., Mladenovic, S., Trifunovic, M., Zdravkovic, M., Manic, M., Trajanovic, M., Misic, D., \& Mitic, J. (2018). Software Framework for the Creation and Application of Personalized Bone and Plate Implant Geometrical Models. Hindawi Journal of Healthcare Engineering. https://doi.org/10.1155/2018/6025935

[26] Ahmadia, M. S., Campolia, G., Yavaria, S. A., Sajadia, B., Wauthle, R., Schrooten, J., Weinansae, H., \& Zadpoora, A. A. (2014). Mechanical behavior of regular open-cell porous biomaterials made of diamond lattice unit cells. Journal of the Mechanical Behavior of BiomedicalMaterials, 34, 106115. https://doi.org/10.1016/j.jmbbm.2014.02.003
Prof. Miroslav TRAJANOVIĆ, PhD

University of Niš,

Faculty of Mechanical Engineering,

Aleksandra Medvedeva 14

18000 Niš, Republic of Serbia

E-mail: miroslav.trajanovic@masfak.ni.ac.rs

Mohammed RASHID, PhD

University of Al- Muthna,

Faculty of Mechanical Engineering,

66001 Samawah, Republic of Iraq

E-mail: miki_plast@yahoo.com

Aleksa MILOVANOVIĆ, MSc

University of Belgrade,

Innovation Center of the Faculty of Mechanical Engineering,

Kraljice Marije 16

11120 Beograd, Republic of Serbia

E-mail: amilovanovic@mas.bg.ac.rs

\section{Contact information:}

\section{Karim Najm HUSAIN, MSc}

University of Al- Qadisiyah,

Faculty of Mechanical Engineering,

58001 Al Diwaniyah, Republic of Iraq

E-mail: husainkarim2014@gmail.com

Prof. Miloš STOJKOVIć, PhD

University of Niš,

Faculty of Mechanical Engineering,

Aleksandra Medvedeva 14 ,

18000 Niš, Republic of Serbia

E-mail: miloss@masfak.niac.rs

Nikola VITKOVIĆ, MSC

University of Niš

Faculty of Mechanical Engineering

Aleksandra Medvedeva 14 ,

18000 Niš, Republic of Serbia

E-mail: nvitko@gmail.com

Jelena MILOVANOVIĆ, MSC

University of Niš,

Faculty of Mechanical Engineering,

Aleksandra Medvedeva 14,

18000 Niš, Republic of Serbia

E-mail: jeki@masfak.ni.ac.rs 